\title{
Curcumin nanoparticles are a promising anti-bacterial and anti-inflammatory agent for treating periprosthetic joint infections
}

This article was published in the following Dove Medical Press journal: International Journal of Nanomedicine

\author{
Kuo-Ti Peng, ${ }^{1,2}$ Yao-Chang \\ Chiang, ${ }^{3,4}$ Tsung-Yu Huang, ${ }^{5,6}$ \\ Pei-Chun Chen,' Pey-Jium \\ Chang, ${ }^{6,7}$ Chiang-Wen Lee ${ }^{3,4,8,9}$ \\ 'Department of Orthopaedic Surgery, \\ Chang Gung Memorial Hospital, Puzi City, \\ Chiayi County 61363, Taiwan; ${ }^{2}$ College of \\ Medicine, Chang Gung University, Guishan \\ District, Taoyuan City 33303, Taiwan; \\ ${ }^{3}$ Department of Nursing, Chang Gung \\ University of Science and Technology, \\ Puzi City, Chiayi County 61363, Taiwan; \\ ${ }^{4}$ Division of Basic Medical Sciences, and \\ Chronic Diseases and Health Promotion \\ Research Center, Chang Gung University \\ of Science and Technology, Puzi City, \\ Chiayi County 61363, Taiwan; ${ }^{5}$ Division \\ of Infectious Diseases, Department of \\ Internal Medicine, Chang Gung Memorial \\ Hospital, Chiayi, Taiwan; ${ }^{6} \mathrm{G}$ raduate Institute \\ of Clinical Medical Sciences, College of \\ Medicine, Chang-Gung University, Taoyuan, \\ Taiwan; ${ }^{7}$ Department of Nephrology, Chang \\ Gung Memorial Hospital, Chiayi, Taiwan; \\ ${ }^{8}$ Research Center for Industry of Human \\ Ecology and Research Center for Chinese \\ Herbal Medicine, Chang Gung University \\ of Science and Technology, Guishan \\ District, Taoyuan City 33303, Taiwan; \\ ${ }^{9}$ Department of Rehabilitation, Chang \\ Gung Memorial Hospital, Puzi City, Chiayi \\ County 61363, Taiwan
}

Correspondence: Chiang-Wen Lee Department of Nursing, Chang Gung University of Science and Technology, Puzi City, Chiayi County 61363, Taiwan

Tel +88653628800 ext 2620

Fax +88653628866

Email cwlee@mail.cgust.edu.tw

Kuo-Ti Peng

Department of Orthopaedic Surgery, Chang

Gung Memorial Hospital, Puzi City, Chiayi

County 61363, Taiwan

Tel +8865362 1000 ext 2004

Email mr3497@cgmh.org.tw
Background: Periprosthetic joint infections (PJIs) have a high incidence of recurrence after total joint replacement and are difficult to treat by debridement or antibiotic treatment. Curcumin is a natural product with anti-inflammatory and anti-bacterial properties. The low bioactivity of curcumin in water restricts its clinical application. Curcumin nanoparticles (CURN) were developed to overcome this limitation.

Methods: In this study, the therapeutic effects of CURN and their anti-inflammatory functions were investigated in a Staphylococcus aureus biofilm-induced PJIs model.

Results: CURN first attenuated the biofilm-induced expansion of myeloid-derived suppressor cells (MDSCs) and then regulated M1- and M2-phenotypic MDSC expression. Down-regulation of cytokines and reactive oxygen species was considered as the mechanism of CURN in reversing the suppression of $\mathrm{T}$ cell proliferation. The recovery of bone permeative destruction demonstrated that CURN enhanced therapeutic potency of vancomycin in vivo.

Conclusion: This is the first study to demonstrate that CURN may be useful for treating PJIs. Keywords: periprosthetic joint infections, osteomyelitis, Staphylococcus aureus, myeloidderived suppressor cells

\section{Introduction}

Joint replacement is the most common elective orthopedic surgeries, which improves the lives of millions of people worldwide. ${ }^{1}$ According to statistical data from 2010, 332,000 total hip and 719,000 total knee arthroplasties were performed in the United States, ${ }^{1}$ and the predicted numbers of total hip and total knee arthroplasties may increase to $137 \%$ and $601 \%$, respectively, reaching 572,000 and $3,480,000$ by $2030 .^{2}$

Periprosthetic joint infections (PJIs) are a huge financial burden for individual patients and the global health care industry. ${ }^{1,3-5}$ The incidence of PJIs is $1 \%-2 \%$ for primary total joint arthroplasties and $2 \%-6 \%$ for revision joint arthroplasties. ${ }^{6}$ Although treatment strategies such as radical debridement with retention of the implants or one-stage (or immediate) or two-stage (or delayed) revisions and excision arthroplasty are applied in the clinic, ${ }^{7,8}$ the treatment failure rate of PJIs remains high at $30 \%-50 \%$ after surgical debridement and over $10 \%$ after one-stage or two-stage surgical treatment in follow-up. ${ }^{9-14}$

PJIs and osteomyelitis (OM) are typically caused by Staphylococcus aureus (S. aureus), a Gram-positive microorganism, ${ }^{14,15}$ which is a major pathogen that causes community-associated nosocomial infections. ${ }^{16,17}$ The biofilm produced by $S$. aureus, a self-produced matrix of hydrated extracellular polymeric substances composed of polysaccharides, proteins, lipids, and extracellular nucleic acids, can contaminate 
internal medical devices and orthopedic implants and surgeries, resulting in serious health care concerns because of their resistance to antibiotics. ${ }^{18}$ Additionally, methicillinresistant $S$. aureus (MRSA) has gradually increased in proportion among the clinical isolates of $S$. aureus,${ }^{19}$ showing $>50 \%$ prevalence among hospital pathogens in several Asian countries ${ }^{20}$ and increases in the human population. ${ }^{21}$ Thus, MRSA is a serious therapeutic problem, ${ }^{21}$ and treatment of $S$. aureus and MRSA-related biofilm infections are an urgent issue for PJIs and public health. ${ }^{14-17,20-22}$

Studies of cellular pathologic changes have suggested that myeloid-derived suppressor cells (MDSCs) and M2-macrophages are involved in $S$. aureus biofilm-infected PJIs. ${ }^{23,24}$ Previously, we established a $S$. aureus biofilminfected animal model to simulate PJIs, and further demonstrated that $S$. aureus biofilm-induced bone marrow cells (BMCs) preferentially promoted the expansion of monocytic but not granulocytic MDSCs, as well as increased the immunosuppressive activity of T cells of overall MDSCs and conversion of monocytic MDSCs into M2-macrophages in vitro and in vivo. ${ }^{25}$ Hence, drugs that influence $S$. aureus biofilm-mediated MDSCs change are candidate agents for treating PJIs.

Conventional curcumin (CUR), a nature polyphenol product, was first identified in 1910 in the rhizome of the medicinal plant Curcuma longa. ${ }^{26}$ Several studies demonstrated that CUR has potential anti-oxidant, anti-inflammatory, anti-bacterial, and anti-tumour growth activities. ${ }^{27-30}$ In cells, CUR regulates the expression of multiple enzymes, kinases, cytokines, growth factors, and transcription factors. ${ }^{31-33}$ Although CUR has numerous cellular functions, it shows low bioavailability which may attributable to its low aqueous solubility and dissolution properties. ${ }^{27,34}$ In general, dimethyl sulfoxide (DMSO) is a useful solvent for solubilising poorly soluble drugs in biochemical and animal experiments. Unfortunately, DMSO is toxic toward normal cells and been rarely used as a solvent in clinical applications. ${ }^{35-38}$ In recent years, nanoparticle formations have been developed to improve the solubility of CUR to increase its bioavailability and pharmacological effects. ${ }^{27,28,34,39}$ A polyvinylpyrrolidone (PVP)-based novel curcumin nanoparticle (CURN) system was developed in our previous study. ${ }^{34}$ The physicochemical properties (including the reduction of particle size and formation of a high-energy amorphous state) of CURN promoted its water solubility and drug release. ${ }^{34}$ Furthermore, CURN improved cellular functions, such as by enhancing anti-oxidant and anti-cancer activities in hepatocytes ${ }^{34}$ and reducing tumour necrosis factor-alpha (TNF- $\alpha$ )-induced in tercellular adhesion molecule-1 expression in human lung epithelial cells as compared to the CUR water preparation. ${ }^{39}$

In the current study, we utilized the $S$. aureus biofilminfected model to evaluate the therapeutic function of CURN. The anti-inflammatory effects of CURN on S. aureus biofilm-induced changes in MDSCs, M1- and M2-phenotypic MDSCs, cytokine expression, T-cell proliferation, and recovery of osteolytic lesion were investigated.

\section{Materials and methods Materials}

The MRSA stain of S. aureus (ATCC43300) was purchased from the Bioresource Collection and Research Center (Hsinchu, Taiwan). CURN was provided by Feng-Lin Yen (Kaohsiung Medical University). The preparation methods were described in previously. ${ }^{34}$ CURN was powdered and stored in a black bottle at $-20^{\circ} \mathrm{C}$. CURN was dissolved in PBS before the experiments were performed. RPMI 1640 medium was obtained from Thermo Fisher Scientific (Waltham, MA, USA). Brain heart infusion (BHI) was obtained from Bacto (Detroit, MI, USA).

\section{Curcumin nanoparticle synthesis}

The synthesis protocol of PVP-based CURN was developed in a previous study. ${ }^{34}$ Briefly, $50 \mathrm{mg}$ of curcumin was dissolved in $25 \mathrm{~mL}$ of ethanol. The $75 \mathrm{~mL} 300 \mathrm{mg}$ PVP contained aqueous solution were quickly injected into the organic phase which was isolated from the curcumin ethanol solution. This mixed solution was homogenized at 22,000 rpm for 25 minutes during the injection process. After that, excess ethanol was completely removed from the mixed solution by rotary vacuum evaporation at $40^{\circ} \mathrm{C}$. The remaining fraction, which consists of the nanoparticle of curcumin, was lyophilized and stored until use.

\section{Animals}

Male C57BL/6 mice (National Laboratory Animal Center, Taipei, Taiwan), weighing 22-30 g, were acclimatized to a room with a controlled temperature $\left(25^{\circ} \mathrm{C}\right)$ and humidity $(50 \% \pm 10 \%)$ with a 12 -hour day-night cycle (light on 07:00-19:00 hours) for 24 hours before performing the experiments. After surgery, the mice were kept individually in separate cages and provided with food (LabDiet 5053; PMI Nutrition International, St Louis, MO, USA) and water ad libitum. Infection with $S$. aureus biofilm was performed on mice aged 12 weeks. Ethical guidelines established by the Chang Gung Memorial Hospital Animal Care were followed throughout the study protocol. The animal studies were 
approved by Institutional Animal Care and Use Committee of Chang Gung Memorial Hospital (IACUC number: 2015120302 and 2016122119).

\section{S. aureus and biofilm preparation}

The $S$. aureus bacteria were cultured in BHI media (Bacto) for 4 days to form the biofilm as previously described. ${ }^{25,40}$ Briefly, the biofilm was collected by centrifugation at $3,900 \times g$ for 15 minutes, and then sterilized by autoclaving or pre-treatment with vancomycin $(20 \mu \mathrm{g} / \mathrm{mL})$ and UV for 12 hours. The pellets of biofilm were redissolved in RPMI 1640 medium (Thermo Fisher Scientific) for in vitro experiments and the concentration was measured using the bicinchoninic acid assay (Thermo Fisher Scientific). For experimental use, S. aureus was pelleted, washed three times with cooled PBS, and resuspended in cooled PBS until use. The concentration of $S$. aureus was estimated by spectrophotometry at an absorbance wavelength of $600 \mathrm{~nm}\left(\mathrm{~A}_{600}\right)$.

\section{Isolation of specific cell populations from mice bone marrow}

BMCs were collected from the bone marrow of the mouse femur. The MDSC population $\left(\mathrm{CD} 11 \mathrm{~b}^{+} \mathrm{Gr} 1^{+}\right)$was sorted from the CD11b-FITC, Gr1-PE double-positive population by using a FACSAria Fusion cell sorter (BD Biosciences, Franklin Lakes, NJ, USA). The purity of the isolated cell population was verified by flow cytometry.

\section{Coculture of isolated cell populations with S. aureus biofilm and CURN in vitro}

Mouse BMCs were seeded into a 24-well plate and cocultured with $0.2 \mathrm{mg} / \mathrm{mL} S$. aureus biofilm loaded in a transwell cell culture insert ( $0.4 \mu \mathrm{m}$ pore size) and treated with different concentrations of CURN. All cell populations were cultured in RPMI 1640 (Thermo Fisher Scientific) containing $10 \%$ FBS. After 48 hours, the cells were analyzed by flow cytometry (BD FACSCanto II) using specific antibodies.

\section{Flow cytometry analysis}

For flow cytometry analysis, $50 \mu \mathrm{L}$ blood samples were collected to determine the dynamic changes in the immune cell populations in the peripheral blood of mice. After lysing the red blood cells, the remaining leukocytes were resuspended in PBS containing $2 \% \mathrm{FBS}$, and then stained with CD11b-FITC (BD Biosciences) or Gr-1-PE (BD Biosciences) for 30 minutes at $4{ }^{\circ} \mathrm{C}$. Mouse cells were analyzed by staining with CD11b-FITC (BD Biosciences), Gr-1-PE (BD Biosciences), CD80-APC (\#104714; Biolegend, San Diego, CA, USA), and dectin-1-APC (eBioscience; Thermo Fisher Scientific). Flow cytometry data from the BD FACSCantoII flow cytometer were evaluated using FACSDiva software (BD Biosciences). The number of events analyzed was 10,000 per sample. Analysis was performed using FlowJo software (Tree Star, Ashland, OR, USA).

\section{Measurement of cytokines in cell culture supernatants}

Cell culture supernatants of mouse BMC with/without $S$. aureus biofilm treated with different concentrations of CURN for 48 hours were used for measuring the levels of cytokines. Cytokines were detected with a BD Cytometric Bead Array Mouse Inflammation kit (\#552364; BD Biosciences) according to the manufacturer's instructions. Briefly, $50 \mu \mathrm{L}$ of the cell culture supernatant was prepared and mixed with capture beads and mouse inflammation PE detection reagent, and then washed with wash buffer. Flow cytometry data from the BD FACSCantoII flow cytometer were acquired using FACSDiva software (BD Biosciences).

\section{Measurement of ROS production}

Reactive oxygen species (ROS) production from mouse BMC with/without $S$. aureus biofilmtreated with different concentrations of CURN for 48 hours were measured using 2',7'-dichlorofluorescin diacetate (DCFDA) (D6883; SigmaAldrich Co., St Louis, MO, USA). The cells were incubated at $25^{\circ} \mathrm{C}$ for 30 minutes in $20 \mu \mathrm{M}$ DCFDA. After incubation, the cells were washed twice with PBS and analyzed by flow cytometry (BD FACSCanto II).

\section{T-cell proliferation assays}

Mice BMCs were seeded into 24-well plates with untreated or $S$. aureus biofilm treated with different concentrations of CURN for 48 hours. The isolated MDSC populations (CD11b/ Gr1 double-positive cells) were subsequently cocultured with 5(6)-carboxyfluorescein N-hydroxysuccinimidyl ester-labeled spleen T cells stimulated by Dynabeads Mouse T-Activator CD3/CD28 (Thermo Fisher Scientific) for 48 hours. The ratio of the tested cell populations to $\mathrm{T}$ cells $\left(2 \times 10^{5}\right.$ cells/well in a 96-well round bottom plate in RPMI 1640 with 10\% FBS) was $0.25: 1$ in the study. To isolate spleen T cells, spleens were gently pressed through a strainer using the plunger end of a syringe to obtain single cells. These cells were washed with PBS buffer and ACK lysing buffer (Thermo Fisher Scientific). Cells in warm RPMI ( $2 \mathrm{~mL}$ ) were injected into the nylon wool column and washed with RPMI. The column was sealed and 
incubated at $37^{\circ} \mathrm{C}$ and $5 \% \mathrm{CO}_{2}$ for 45 minutes. Cells were then eluted with $10 \mathrm{~mL}$ of warm RPMI medium.

\section{S. aureus biofilm infection in mice}

Male C57BL/6J mice were anesthetised with 50\% Zoletil $(1 \mathrm{~mL} / \mathrm{kg}$ ) and $2 \%$ rompun mixture solution. The skin near the right knee was disinfected with povidone-iodine and then an incision was made. A 26-gauge needle was utilized to make a burr hole in the femoral intercondylar notch extending into the intramedullary canal, and then a pre-cut $0.5 \mathrm{~cm}$ orthopedic grade Kirschner (K) wire (0.6 mm diameter; Synthes GmbH, Solothurn, Switzerland) was implanted into the intramedullary canal. The $S$. aureus $\left(2 \times 10^{3} \mathrm{CFU}\right)$ biofilm was inoculated into the intramedullary canal containing the implant, the burr hole was sealed with bone wax, and the surgical site was closed with coated Vicryl* 4-0 sutures (Ethicon Inc., Bridgewater, NJ, USA). Sterile implants were used for the sham-operated group. Ketorolac $(0.5-1 \mathrm{mg} / \mathrm{kg}$, intramuscularly) was used to treat the mice immediately after surgery and 24 hours later for the PJI model to evaluate relief from pain. The $\mathrm{C} 57 \mathrm{BL} / 6 \mathrm{~J}$ mice were divided into seven groups, including the sham group and six different treated groups ( $n=4$ for each group). The six experimental groups were the following: 1) the group administered CURN (20 mg/kg) only; 2) the group infected with $S$. aureus only; 3 ) the group injected intraperitoneally with vancomycin $(2 \mathrm{mg} / \mathrm{kg})$ only after infection with $S$. aureus for 7 days; 4-6) the groups injected intraperitoneally with different concentrations of CURN (5, 10, and $20 \mathrm{mg} / \mathrm{kg}$ in PBS, respectively) and vancomycin (2 mg/kg) every day after infection with $S$. aureus for 7 days. Mice containing sterile implants served as the sham-operated group. At 2 weeks after injection, the mice were sacrificed and the femurs were fixed in $10 \%$ formalin.

\section{Data analyses and statistics}

All data were evaluated and graphed with Microsoft Excel software (Microsoft, Redmond, WA, USA). The results were expressed as the mean $\pm \mathrm{SD}$. The results were tested using one-way ANOVA with the post-hoc Tukey's correction and GraphPad Prism software (GraphPad, San Diego, CA, USA). A $P$-value $<0.05$ was considered significant.

\section{Results}

\section{Effects of CURN on S. aureus biofilm- induced BMCs in regulating MDSC \\ expansion}

We examined whether CURN affects $S$. aureus biofilm infection-induced expansion of BMCs to MDSCs in the PJI model.
For establishing the biofilm-stimulated BMC system, several methods were tested, which included autoclaving and pretreatment with antibiotic or UV for 12 hours. Results showed that pre-treatment with antibiotic or UV failed to reduce the $S$. aureus growth rate compared to sterilization by using an autoclave; thus, autoclaving method was found to be the optimal method in our system (Figure S1). A previous study has demonstrated that even after autoclaving, biofilm still shows stimulated effects on BMC cells. ${ }^{25}$ As shown in Figure 1A, after treating the mouse BMCs with $S$. aureus biofilm for 48 hours, the expansion of MDSCs was increased by nearly 1.6 -fold (41.6\% without and $62.6 \%$ with biofilm exposure). After adjusting for each control group, CURN notably suppressed biofilm-induced MDSC expansion in a dose-dependent manner (Figure 1B), while no effects were observed in the non-biofilm treatment groups. Interestingly, CUR dissolved in PBS did not affect MDSC expansion in the biofilm or non-biofilm groups (Figure 1C). This indicates the CURN, which has higher water solubility, effectively reduced biofilm-induced MDSC expansion but did not change the normal MDSC expansion rate under non-infected state.

\section{Effects of CURN on the conversion of MDSCs to MI- and M2-phenotypic MDSCs upon exposure to $S$. aureus biofilm}

Because M1 and M2 cells play different roles, we next evaluated the effects of CURN on M1- and M2-phenotypic MDSC expression with/without biofilm treatment. In general, M1-phenotypic MDSCs play a pro-inflammatory role, while anti-inflammatory M2-phentotypic MDSCs are involved in adaptive immunity and the inflammatory circuit. ${ }^{41,42}$ As shown in Figure 2A, CURN slightly but effectively decreased M1-phenotypic marker expression after biofilm exposure. In contrast, biofilm-induced M2-phenotypic marker expression was enhanced by treatment with CURN in a dose-dependent manner (Figure 2B). This result suggests a prominent anti-inflammatory effect of CURN as a result of S. aureus biofilm infection.

\section{Effects of CURN on S. aureus biofilm- induced inflammatory factors}

Several inflammatory factors, such as IL-6, TNF- $\alpha$, and monocyte chemoattractant protein-1 (MCP-1), are highly associated with PJIs. ${ }^{43,44}$ Thus, the levels of these proteins were evaluated after CURN treatment. CURN dosedependently reduced the expression of biofilm-induced IL-6 

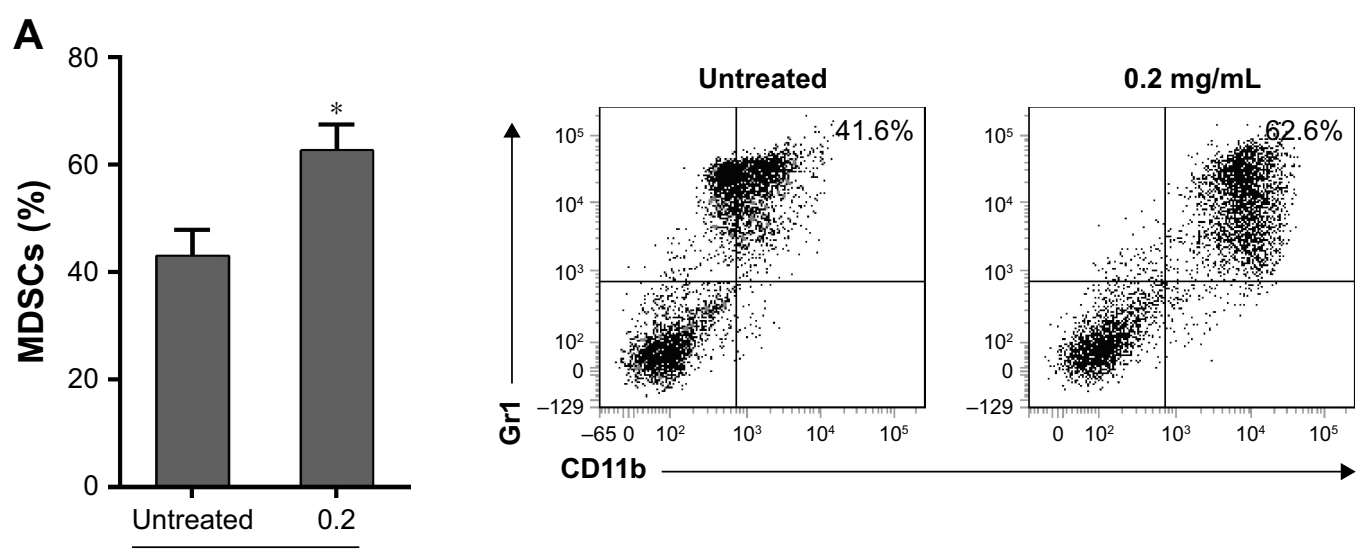

Biofilm (mg/mL)
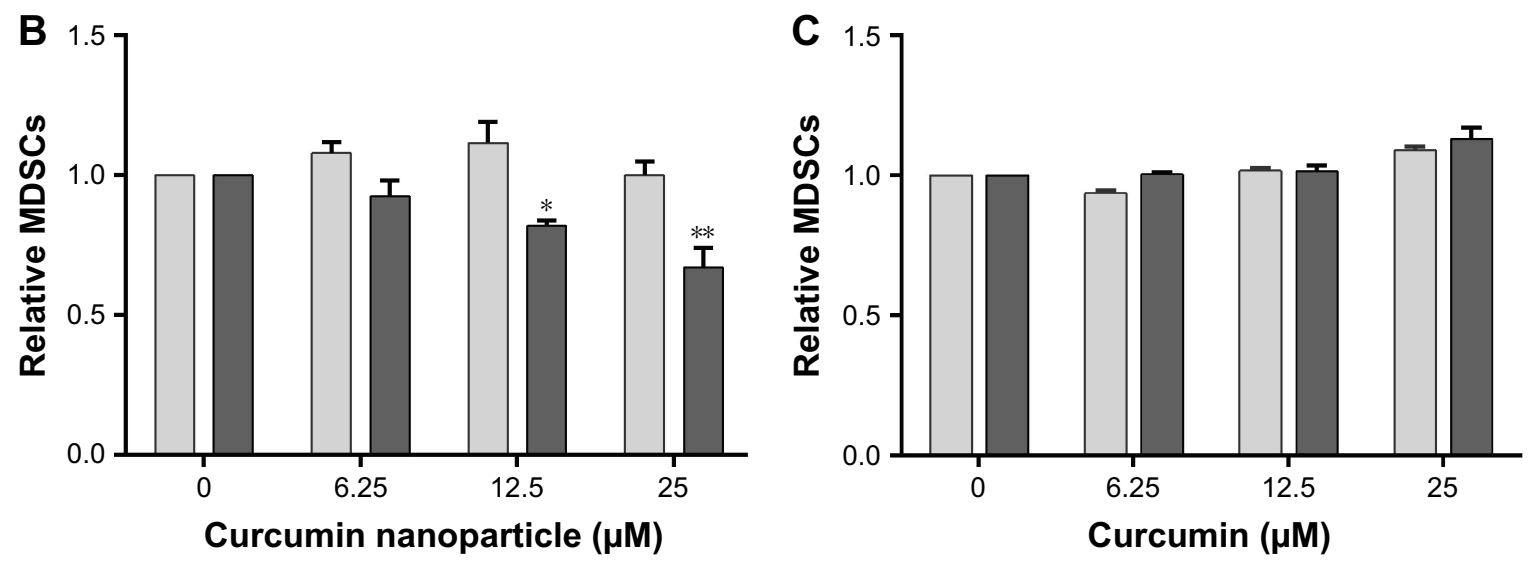

$\square$ Untreated $\square 0.2 \mathrm{mg} / \mathrm{mL}$ biofilm

Figure I (A) MDSC expression in S. aureus biofilm exposure. The bar graph (left panel) was illustrated and converted based on the flow cytometry results (right panel). (B) CURN suppressed biofilm-induced MDSC expansion in a dose-dependent manner. (C) CUR dissolved in PBS did not affect MDSC expansion. Data are expressed as the mean $\pm S D, * P<0.05$ compared to biofilm-untreated group in $(\mathbf{A})$. $* P<0.05$ and $* * P<0.01$ compared to the biofilm-induced but no CURN treated group. Abbreviations: CUR, curcumin; CURN, CUR nanoparticles; MDSCs, myeloid-derived suppressor cells; S. aureus, Staphylococcus aureus.
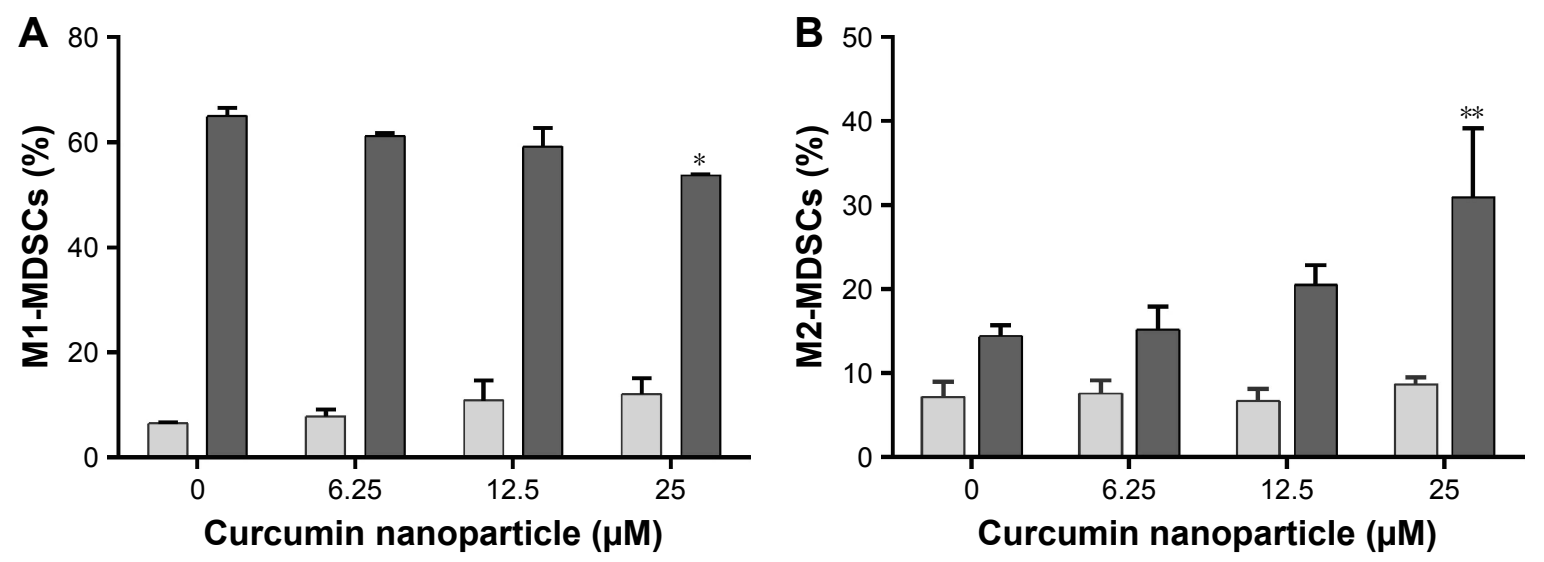

$\square$ Untreated $\square 0.2 \mathrm{mg} / \mathrm{mL}$ biofilm

Figure 2 CURN induced expansion of MDSCs to MI- (A) and M2-phenotypic (B) MDSCs upon exposure to S. aureus biofilm via its anti-inflammatory activity. Notes: Data are expressed as the mean \pm SD. $* P<0.05$ and $* * P<0.01$ compared to the biofilm-induced but no CURN treated group. Abbreviations: CURN, curcumin nanoparticles; MDSCs, myeloid-derived suppressor cells; S. aureus, Staphylococcus aureus. 
A

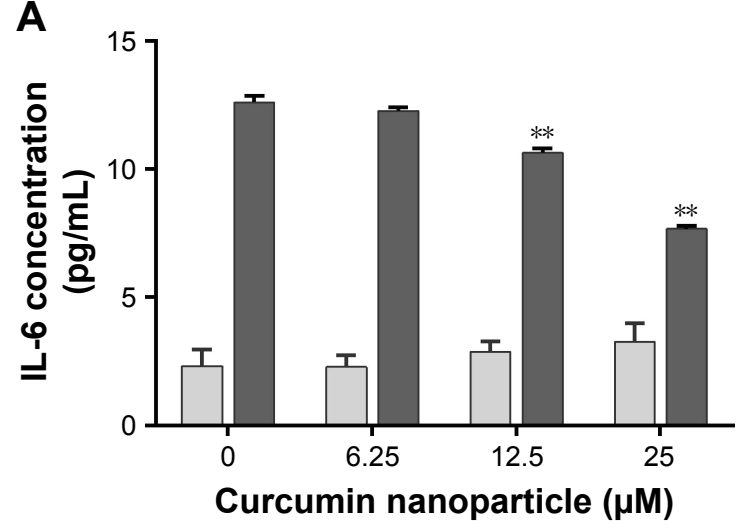

B

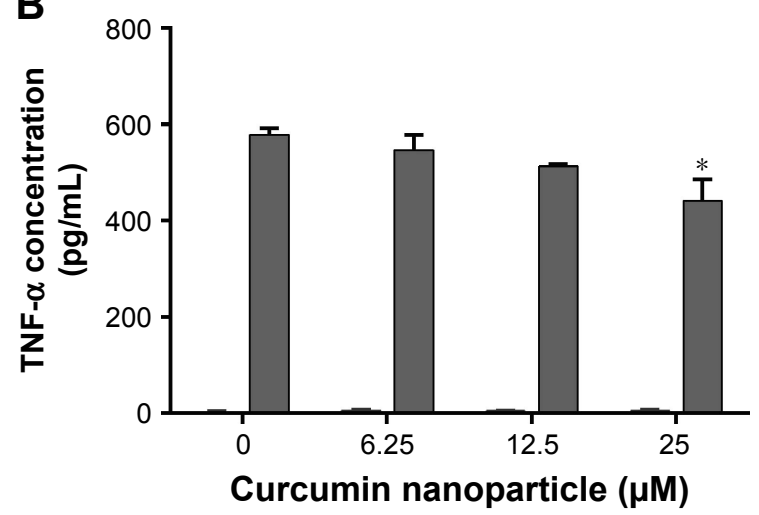

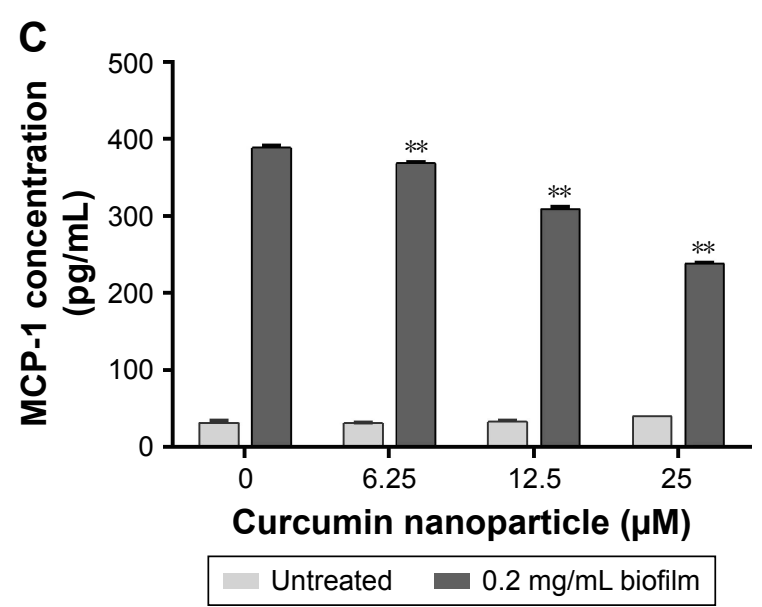

Figure 3 Cytokines including IL-6 (A), TNF- $\alpha(\mathbf{B})$, and MCP-I (C) were downregulated by CURN in the mouse BMC culture supernatants of S. aureus biofilm-stimulated MDSCs.

Notes: Data are expressed as the mean $\pm S D$. $* P<0.05$ and $* * P<0.01$ compared to the biofilm-induced but no CURN treated group.

Abbreviations: BMCs, bone marrow cells; CURN, curcumin nanoparticles; MCP-I, monocyte chemoattractant protein-I; MDSCs, myeloid-derived suppressor cells; TNF- $\alpha$, tumour necrosis factor-alpha; S. aureus, Staphylococcus aureus.

(39.2\% at $25 \mu \mathrm{M}), \mathrm{MCP}-1(38.7 \%$ at $25 \mu \mathrm{M})$, and TNF- $\alpha$ ( $18 \%$ at $25 \mu \mathrm{M})$, while no significant effects were observed in the biofilm-negative groups (Figure 3A-C). Additionally, ROS play a key signaling role in the progression of inflammatory disorders. ${ }^{45}$ Biofilm caused increases in ROS, which were also dose-dependently suppressed by CURN. At the highest CURN dose $(25 \mu \mathrm{M})$, ROS production was notably suppressed by more than $50 \%$ (Figure 4 ). These results suggest that CURN can reverse the inflammatory status triggered by $S$. aureus biofilm.

\section{Effects of CURN on S. aureus biofilm- treated MDSC and T-cell proliferation}

T cells play important roles in anti-bacterial infections. ${ }^{46,47}$ It is well-known that MDSCs are potent inhibitors of T-cell activation in $S$. aureus infection. ${ }^{48-50}$ We previously showed that $S$. aureus biofilm increased MDSC expansion and subsequent T-cell suppression. ${ }^{25}$ Compared to non-MDSC controls, coculture with MDSCs decreased T-cell proliferation by
$41.4 \%$, while treatment with biofilm reduced the proliferation by up to $62.8 \%$ (Figure 5). CURN reversed T-cell proliferation in a dose-dependent manner. The reversal effects of CURN on MDSCs suppressed T-cell proliferation not only in the biofilm-exposed groups but also in the control groups. However, the recovery rate rapidly increased in the biofilm groups. This result indicates that CURN effectively recovered the biofilm-mediated decrease in T-cell activity.

\section{Biofilm formation in a S. aureus-infected PJI model}

To evaluate whether CURN is useful as a therapeutic agent for treating PJIs, in vivo analysis was performed. A mouse PJI model, which we established previously, ${ }^{25}$ was utilized to mimic $S$. aureus biofilm infection in vivo. As shown in Figure 6, sham-operated and CURN-treated mice showed no changes in bone formation according to micro-computed tomography (CT) images. Bone in the S. aureus biofilm infection groups showed severe permeated pathologic 


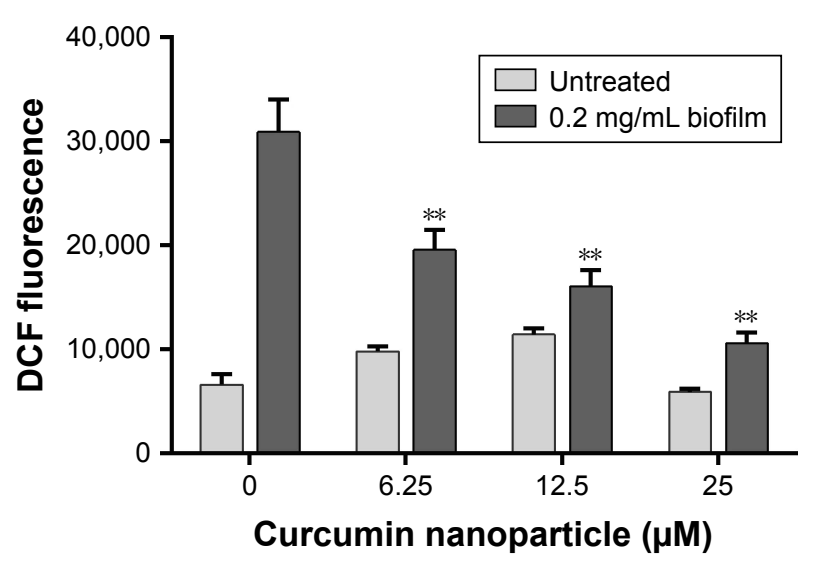

CURN $(\mu \mathrm{M}) \quad 0$

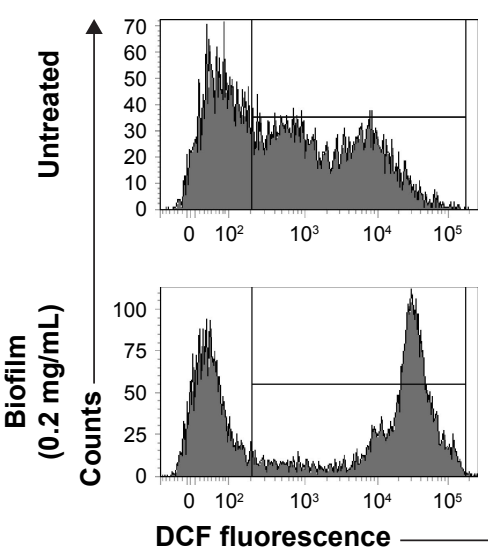

6.25
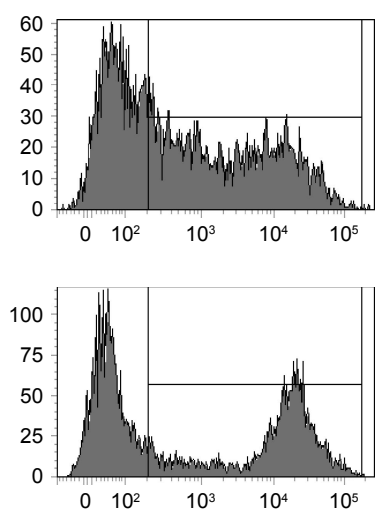

12.5
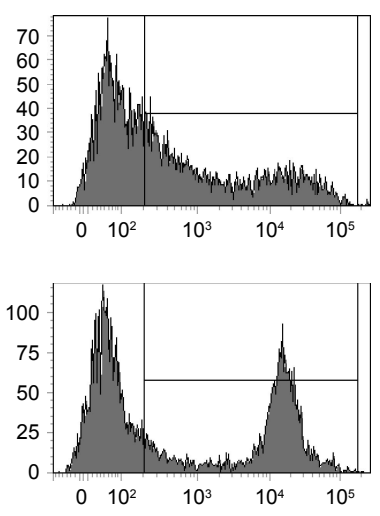

25
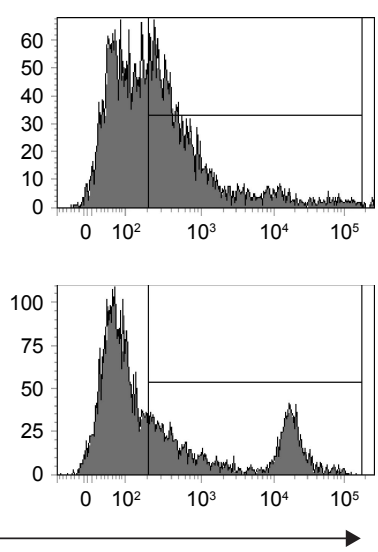

Figure 4 ROS generation from biofilm induced in mouse BMCs was suppressed by CURN in vitro.

Notes: $D C F$ fluorescence was measured by flow cytometry. The bar graphs illustrate the signals of $D C F$ fluorescence. Data are expressed as the mean $\pm S D$. $* * P<0.0$ I compared to the biofilm-induced but no CURN treated group.

Abbreviations: BMCs, bone marrow cells; CURN, curcumin nanoparticles; DCF, dichlorofluorescin; ROS, reactive oxygen species.

\section{T-cell cocultured with CURN- treated MDSCs}

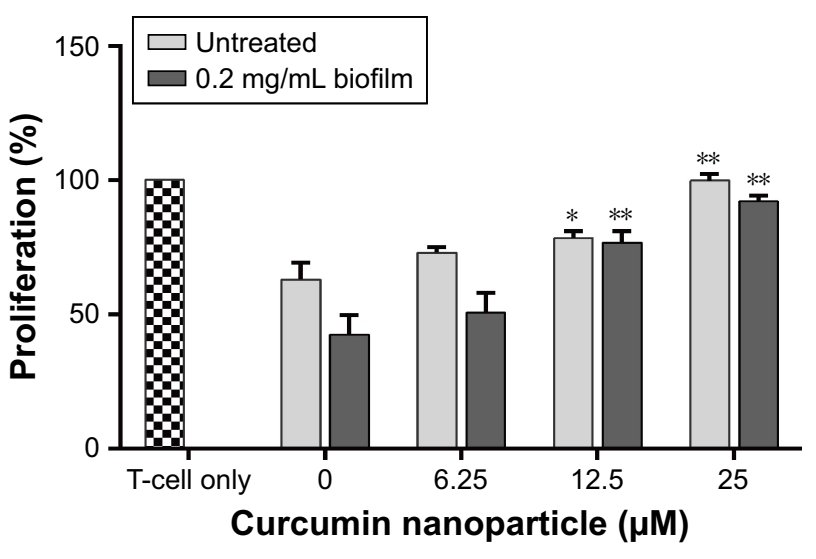

Figure 5 CURN reversed the immunosuppressive activity of $S$. aureus biofilmtreated MDSCs on T cells.

Notes: The differentiation rate of naive $T$ cells after receiving CD3/CD28 beads stimulation was set as $100 \%$ (the mosaic bar). The changes in T-cell differentiation rate were measured after coculturing with MDSCs, which were pretreated with different concentrations of CURN and biofilm. Data are expressed as the mean $\pm S D$. $* P<0.05$ and $* * P<0.01$ compared to the biofilm-induced but no CURN treated group.

Abbreviations: CURN, curcumin nanoparticles; MDSCs, myeloid-derived suppressor cells; S. aureus, Staphylococcus aureus. fracture and was recovered with the general clinical treatment strategy, that is, vancomycin treatment. Osteolytic destructions were lower after cotreatment with CURN and vancomycin compared to the group treated with vancomycin alone, both showing a dose-dependent effect. Furthermore, the structures stained by H\&E staining were consistent with the results of micro-CT imaging (Figure 7). Vancomycin decreased inflammation levels (swollen tissues and broken bones) and lymphocyte expression, but was more effective in reducing inflammation levels and lymphocytes in the vancomycin-CURN cotreated groups. Additionally, to confirm whether the CURN-suppressed MDSC expansion occurred in the PJI in vivo model, blood MDSC levels were measured. The MDSC levels were increased after $S$. aureus biofilm infection (increased by $117.5 \%$ compared to the sham group), while the rate of MDSC expansion was reduced after vancomycin treatment (decreased by $10.3 \%$ compared to the infected group). Cotreatment with vancomycin and CURN notably affected MDSC expansion compared to vancomycin 
A

Sham

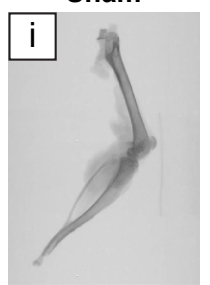

CURN

$20 \mathrm{mg} / \mathrm{kg}$

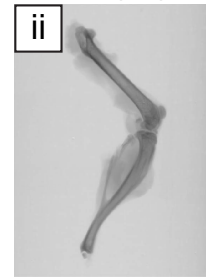

S. aureus

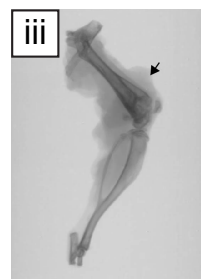

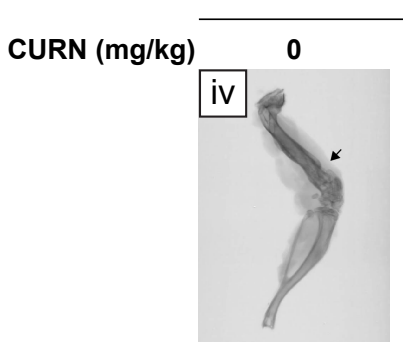

S. aureus + vancomycin 5
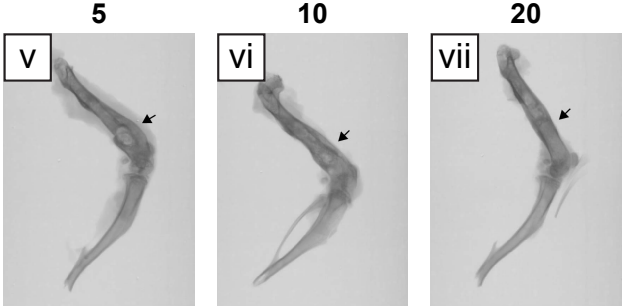

B

Sham

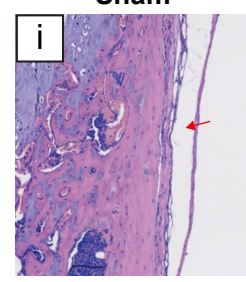

CURN $20 \mathrm{mg} / \mathrm{kg}$

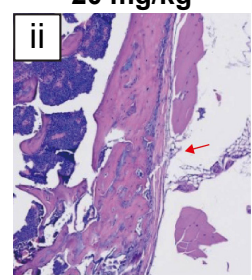

S. aureus
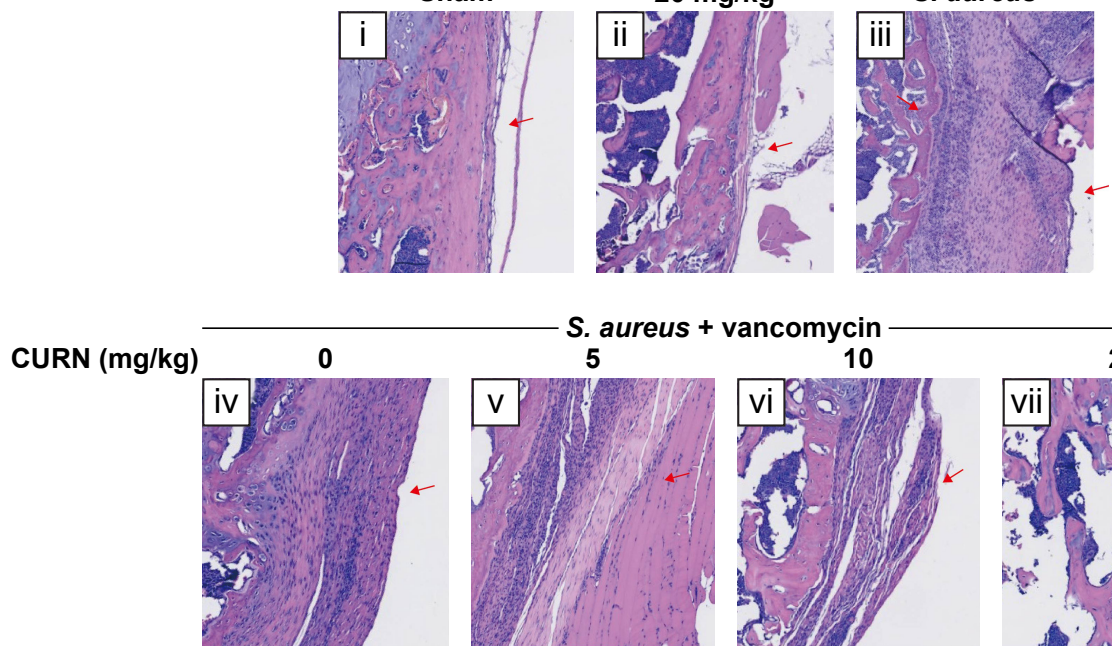

20
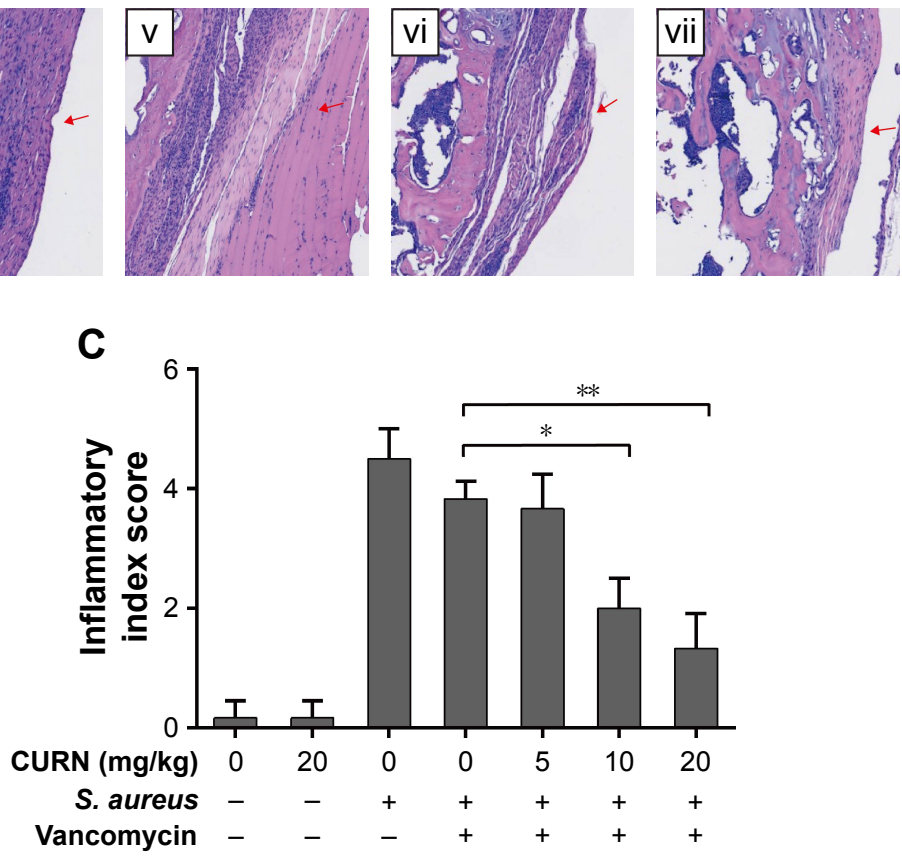

Figure 6 Evaluation of infection level in mouse femur.

Notes: (A) Micro-CT images, in which black arrows indicate the grade of severity in bone destruction: (i) sham group, (ii) CURN only, (iii) permeative destruction with S. aureus implantation, (iv) pathologic fracture after vancomycin $(2 \mathrm{mg} / \mathrm{kg})$ treatment, and ( $\mathrm{v}$-vii) the less osteolytic size in the gradient concentration of CURN. (B) Histological analysis by H\&E staining showed that CURN was effective for treating PJls cotreated with vancomycin ( $2 \mathrm{mg} / \mathrm{kg})$. The labeling of figures is the same as that of micro-CT images. Red arrows indicate infiltrating mononuclear immune cells. (C) The inflammatory index score was evaluated based on the levels of inflammatory cell infiltration in the femur ( $n=3$ for each group). Red arrows indicate infiltrating mononuclear immune cells. Data are expressed as the mean $\pm S D$. $* P<0.05$ and $* * P<0.01$ compared to the group of $S$. aureus infection treated with vancomycin alone.

Abbreviations: CT, computed tomography; CURN, curcumin nanoparticles; PJl, periprosthetic joint infection; S. aureus, Staphylococcus aureus. 


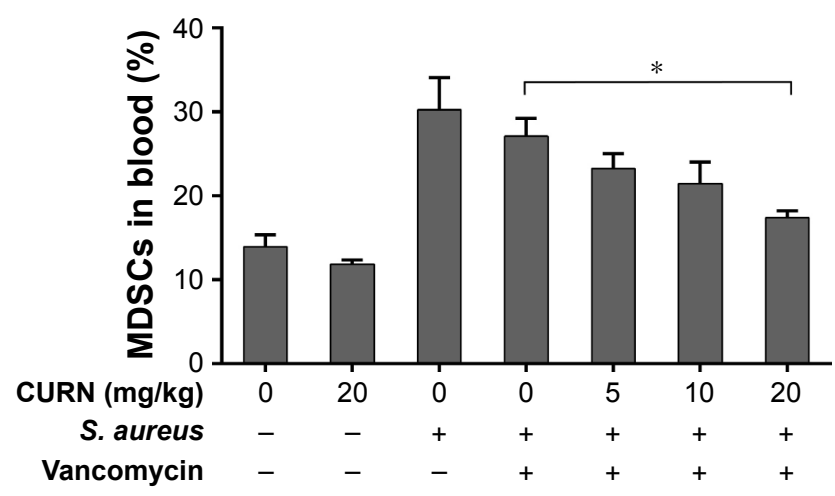

Figure 7 CURN reduces the expansion of MDSCs in the peripheral blood from mice infected with $S$. aureus.

Notes: Data are expressed as the mean \pm SD. $* P<0.05$ compared to the group of S. aureus infection treated with vancomycin $(2 \mathrm{mg} / \mathrm{kg})$ alone.

Abbreviations: CURN, curcumin nanoparticles; MDSCs, myeloid-derived suppressor cells; S. aureus, Staphylococcus aureus.

treatment alone $(5,10$, and $20 \mathrm{mg} / \mathrm{kg}$ CURN: $23.2 \%, 29 \%$, and $42.4 \%$ respectively, decrease compared to the infected group). These results strongly suggest that CURN decreased MDSC expansion and is a potential therapeutic agent for use with antibiotics to treat $S$. aureus biofilm-infected PJIs.

\section{Discussion}

In the current study, we clearly demonstrated that CURN is a useful assistant agent for treating PJIs and OM through in vitro and in vivo experiments. CURN significantly suppressed $S$. aureus biofilm-triggered MDSC expansion and bilaterally regulated the conversion rates of MSDCs to M1- or M2-phenotypic cell expression. This indicates that CURN can reverse the $S$. aureus biofilm-induced immune imbalance. To characterize the possible cellular mechanisms of CURN, we analyzed the levels of PJI-associated inflammatory cytokines and ROS. Our results suggest that CURN notably reduced $S$. aureus biofilm-enhanced IL-6, TNF- $\alpha$, and MCP- 1 concentrations and ROS production in cultured MDSCs. Furthermore, the proliferation value and rate of T-cell suppression by $S$. aureus biofilm infection were recovered by CURN treatment. An in vivo animal PJI model was used to confirm our in vitro findings. The results clearly showed that CURN improved the effects of traditional clinical strategy - vancomycin treatment - on the inflammatory site and structure formation and on MDSCs in the blood.

PJI is an increasing and difficult-to-treat infection caused by $S$. aureus or MRSA biofilm. ${ }^{19-21}$ PJIs show a high risk to progress to chronic OM.,51 The antimicrobial therapy is not very effective in treating bone and joint infections because of the physiological and anatomical characteristics of these infections, and surgical debridement is often necessary. ${ }^{51}$
However, the success rate of treating PJIs is low, with failure rates being in the range $30 \%-50 \%{ }^{9-14}$ The overuse of antibiotics leads to the development of multi-resistant bacteria and harms human health. Thus, natural bioactive compounds may be useful for developing therapeutic agents for treating bacterial infections as alternatives to synthetic drugs.

Conventional CUR is a natural product identified more than 100 years ago. ${ }^{26}$ The systemic functions of CUR include anti-inflammation, anti-cancer, anti-oxidant, bacterial growth suppression, and regulation of gut microbiota composition, ${ }^{27-30,52}$ and CUR plays cellular roles in regulating multiple enzymes, kinases, cytokine growth factors, and transcription factors release. ${ }^{31-33}$ While CUR is a potential antibacterial agent, it is difficult to apply in the clinical setting because of its low bioavailability, solubility, and dissolution properties in water. ${ }^{27,34}$ Owing to the advances in nanochemistry, the potential for using CUR in the clinic has increased.

Since hematopoiesis is altered in chronic infections and cancer, MDSCs are found to expand greatly. ${ }^{53}$ Thus, PVP-based CURN, developed in our previous study, may suppress the expansion to decrease infection levels. Under pathological conditions, several inflammatory factors are involved in MDSC expansion, such as pro-inflammatory cytokines like TNF- $\alpha$, IL-6, prostaglandin E2, etc. ${ }^{49}$ Moreover, IL-6, TNF- $\alpha$, and MCP-1 are involved in inflammation and are sensitive markers for PJIs or deep implants. ${ }^{43,44}$ $S$. aureus biofilm infection induced the upregulation of TNF- $\alpha$, IL-6, and MCP-1, which were reduced by CURN treatment in this study. This suppression agrees with the results of previous studies on inflammation ${ }^{29}$ and indicates that CURN reduced MDSC expansion by downregulating the expression of inflammatory cytokines.

Traditionally, MDSCs can be classified as monocytic (M-MDSC, CD11 b+Ly6C ${ }^{+} \mathrm{Ly} 6 \mathrm{G}^{\text {low }}$ ) and granulocytic (G-MDSC, CD11b+Ly6C ${ }^{\text {low }} \mathrm{L} 6 \mathrm{G}^{+}$) based on the expression levels of Ly6C and Ly6G. ${ }^{49} S$. aureus biofilm infections preferentially promote M-MDSC expansion in PJI models and other infectious diseases. ${ }^{25,54}$ M-MDSCs generated high levels of inducible nitric oxide, which can cause T-cell receptor nitration and prevent its interaction with antigen-major histocompatibility complex. Additionally, high levels of inducible nitric oxide synthase could restrict the availability of amino acid-L-arginine, which is essential for $\mathrm{T}$ cell proliferation, subsequence to suppress $\mathrm{T}$ cell activity. ${ }^{49}$ CUR was also reported to play a role in macrophage polarization. Previous studies showed that CUR inhibited M1 macrophage polarization and TNF- $\alpha$, IL-6, and IL-12B production in a dose-dependent manner ${ }^{55}$ and 
induced M2 macrophage polarization via the secretion of IL-4 and/or IL-13. ${ }^{56}$ Furthermore, M1 macrophages respond to inflammatory signaling by playing a pro-inflammatory role, while M2 macrophages participate in the resolution of the inflammatory process by playing an anti-inflammatory role and produce anti-inflammatory cytokines to facilitate tissue healing. ${ }^{41}$ Our results showed that CURN decreased M1- and increased M2-phenotypic expression, which may improve the anti-inflammatory effects and promote tissue recovery.

The anti-inflammatory effects of CUR or CURN may occur through the reduction of ROS production. ${ }^{34,39}$ Nicotinamide adenine dinucleotide phosphate (NADP) oxidases are ROS generators and have been identified in phagocytes for bacterial killing. ${ }^{45}$ In cancer, tissue damage, and inflammation, ROS production is increased because MDSCs express the NADPH oxidase (Nox2) components $\mathrm{p} 47^{\text {phox }}$ and gp91 ${ }^{\text {phox }}$ and upregulate Nox2 activity. Consistently, deficits in $\mathrm{p} 47^{\text {phox }}$ or Nox 2 protect against damages from TNF- $\alpha$ induced inflammatory response. ${ }^{45}$ In general, MDSCs exhibit strong immunosuppressive activities rather than immunostimulation functions. ${ }^{57}$ Furthermore, suppression of ROS production reverses the immunosuppressive function of MDSCs to T-cell responses. ${ }^{53,58}$ Not only ROS, but also MDSCs inhibit T-cell responses through multiple pathways, such as NO production, amino acid metabolism, and cytokines, among others. A previous study suggested that T-cell suppression of MDSCs is related to cancer-derived IL-6, and inhibition of IL-6-mediated signals improved the prognosis of cancer. ${ }^{59} \mathrm{~T}$-cell function is gradually decreased in $S$. aureus-induced chronic abscess and is associated with infection progression. ${ }^{48} \mathrm{~T}$ cells also kill bacteria during infection. ${ }^{47}$ Thus, reversing the suppression of T-cell function is beneficial for treating PJIs. Our results indicate that $S$. aureus biofilm in MDSCs showed greater immunosuppressive effects on T-cell proliferation, but this may be reversed by CURN treatment, which decreases MDSC-triggered ROS production and cytokine expression. This finding strongly suggests that CURN is useful as an immunoactivity enhancer for treating $S$. aureus biofilm infection-related diseases, such as PJIs or OM.

Local treatment with nano-formulated CUR effectively inhibited inflammation and bone resorption in an experimental periodontal disease model. ${ }^{60}$ This finding and our results following CURN administration in the PJI model suggest that CURN had anti-inflammation effects and improved bone absorption. After cotreatment with vancomycin, CURN recovered $S$. aureus biofilm-induced serious bone destruction, while CUR in PBS was not effective (data not shown). Furthermore, suppression of the expansion of MDSCs by CURN in the blood provides cellular evidence for the recovery of bone destruction.

In conclusion, this is the first study to demonstrate that CURN is useful for treating PJIs based on in vitro and in vivo analysis. CURN reduced cytokine expression and ROS production to suppress $S$. aureus biofilm-induced MDSCs expansion and increased the expression levels of M2-phenotypic cells to reverse the proliferation of $\mathrm{T}$ cells. Furthermore, the permeative destruction caused by $S$. aureus biofilm infection was effectively recovered by cotreatment with CURN and vancomycin. Therefore, CURN can be considered as a potential additive anti-infective agent in the treatment of PJIs and OM.

\section{Acknowledgments}

We thank Feng-Lin Yen (Kaohsiung Medical University) for providing curcumin-loaded PVP nanoparticles for this study. This work was supported by the Chang Gung Medical Research Program Foundation (grant numbers CMRPG6F0341, CMRPG6F0342, CMRPG6G0231, and CMRPG6H0401 from the Chang-Gung Memorial Hospital, Taiwan), and the Ministry of Science and Technology (ROC) (grant numbers NMRPG6G6011 and NMRPG6G6012).

\section{Disclosure}

The authors report no conflicts of interest in this work.

\section{References}

1. Tande AJ, Patel R. Prosthetic joint infection. Clin Microbiol Rev. 2014; 27(2):302-345.

2. Kurtz S, Ong K, Lau E, Mowat F, Halpern M. Projections of primary and revision hip and knee arthroplasty in the United States from 2005 to 2030. J Bone Joint Surg Am. 2007;89(4):780-785.

3. Kurtz SM, Lau E, Watson H, Schmier JK, Parvizi J. Economic burden of periprosthetic joint infection in the United States. J Arthroplasty. 2012;27(8 Suppl):61-65.

4. Peel TN, Dowsey MM, Buising KL, Liew D, Choong PF. Cost analysis of debridement and retention for management of prosthetic joint infection. Clin Microbiol Infect. 2013;19(2):181-186.

5. McConoughey SJ, Howlin R, Granger JF, et al. Biofilms in periprosthetic orthopedic infections. Future Microbiol. 2014;9(8):987-1007.

6. Gallo J, Kolar M, Novotny R, Rihakova P, Ticha V. Pathogenesis of prosthesis-related infection. Biomed Pap Med Fac Univ Palacky Olomouc Czech Repub. 2003;147(1):27-35.

7. Rao N, Ziran BH, Lipsky BA. Treating osteomyelitis: antibiotics and surgery. Plast Reconstr Surg. 2011;127(Suppl 1):177S-187S.

8. Fagotti L, Tatka J, Salles MJC, Queiroz MC. Risk factors and treatment options for failure of a two-stage exchange. Curr Rev Musculoskelet Med. 2018:420-427.

9. Vilchez F, Martínez-Pastor JC, García-Ramiro S, et al. Outcome and predictors of treatment failure in early post-surgical prosthetic joint infections due to Staphylococcus aureus treated with debridement. Clin Microbiol Infect. 2011;17(3):439-444. 
10. Byren I, Bejon P, Atkins BL, et al. One hundred and twelve infected arthroplasties treated with 'DAIR' (debridement, antibiotics and implant retention): antibiotic duration and outcome. J Antimicrob Chemother. 2009;63(6):1264-1271.

11. Bryan AJ, Abdel MP, Sanders TL, Fitzgerald SF, Hanssen AD, Berry DJ. Irrigation and debridement with component retention for acute infection after hip arthroplasty: improved results with contemporary management. J Bone Joint Surg Am. 2017;99(23):2011-2018.

12. Lora-Tamayo J, Murillo O, Iribarren JA, et al. A large multicenter study of methicillin-susceptible and methicillin-resistant Staphylococcus aureus prosthetic joint infections managed with implant retention. Clin Infect Dis. 2013;56(2):182-194.

13. Nodzo SR, Boyle KK, Spiro S, Nocon AA, Miller AO, Westrich GH. Success rates, characteristics, and costs of articulating antibiotic spacers for total knee periprosthetic joint infection. Knee. 2017;24(5): 1175-1181.

14. Ricciardi BF, Muthukrishnan G, Masters E, Ninomiya M, Lee CC, Schwarz EM. Staphylococcus aureus evasion of host immunity in the setting of prosthetic joint infection: biofilm and beyond. Curr Rev Musculoskelet Med. Epub 2018 Jul 9.

15. Gbejuade HO, Lovering AM, Webb JC. The role of microbial biofilms in prosthetic joint infections. Acta Orthop. 2015;86(2):147-158.

16. Jones M, Ying J, Huttner B, et al. Relationships between the importation, transmission, and nosocomial infections of methicillin-resistant Staphylococcus aureus: an observational study of 112 Veterans Affairs Medical Centers. Clin Infect Dis. 2014;58(1):32-39.

17. Keynan Y, Rubinstein E. Staphylococcus aureus bacteremia, risk factors, complications, and management. Crit Care Clin. 2013;29(3):547-562.

18. Flemming HC, Wingender J. The biofilm matrix. Nat Rev Microbiol. 2010;8(9):623-633.

19. Hiramatsu K, Katayama Y, Yuzawa H, Ito T. Molecular genetics of methicillin-resistant Staphylococcus aureus. Int J Med Microbiol. 2002;292(2):67-74.

20. Grundmann H, Aires-de-Sousa M, Boyce J, Tiemersma E. Emergence and resurgence of meticillin-resistant Staphylococcus aureus as a public-health threat. Lancet. 2006;368(9538):874-885.

21. Drago L, De Vecchi E, Nicola L, Gismondo MR. In vitro evaluation of antibiotics' combinations for empirical therapy of suspected methicillin resistant Staphylococcus aureus severe respiratory infections. BMC Infect Dis. 2007;7(1):111.

22. Hanke ML, Heim CE, Angle A, Sanderson SD, Kielian T. Correction: targeting macrophage activation for the prevention and treatment of Staphylococcus aureus biofilm infections. J Immunol. 2013;190(12) 6709-6710.

23. Gabrilovich DI, Nagaraj S. Myeloid-derived suppressor cells as regulators of the immune system. Nat Rev Immunol. 2009;9(3):162-174.

24. Peranzoni E, Zilio S, Marigo I, et al. Myeloid-derived suppressor cell heterogeneity and subset definition. Curr Opin Immunol. 2010;22(2): 238-244.

25. Peng KT, Hsieh CC, Huang TY, et al. Staphylococcus aureus biofilm elicits the expansion, activation and polarization of myeloid-derived suppressor cells in vivo and in vitro. PLoS One. 2017;12(8):e0183271.

26. Miłobędzka J, Kostanecki SV, Lampe V. Zur Kenntnis des Curcumins [Structure of curcumin]. Berichte der Deutschen Chemischen Gesellschaft. 1910;43(2):2163-2170. German.

27. Gera M, Sharma N, Ghosh M, et al. Nanoformulations of curcumin: an emerging paradigm for improved remedial application. Oncotarget. 2017;8(39):66680-66698.

28. Yallapu MM, Jaggi M, Chauhan SC. Curcumin nanoformulations: a future nanomedicine for cancer. Drug Discov Today. 2012;17(1-2): 71-80.

29. Chainani-Wu N. Safety and anti-inflammatory activity of curcumin: a component of tumeric (Curcuma longa). J Altern Complement Med. 2003;9(1):161-168.

30. Li B, Li X, Lin H, Zhou Y. Curcumin as a promising antibacterial agent: effects on metabolism and biofilm formation in S. mutans. Biomed Res Int. 2018;2018(3):1-11.
31. Jin CY, Lee JD, Park C, Choi YH, Kim GY. Curcumin attenuates the release of pro-inflammatory cytokines in lipopolysaccharide-stimulated BV2 microglia. Acta Pharmacol Sin. 2007;28(10):1645-1651.

32. Yadav R, Jee B, Awasthi SK. Curcumin suppresses the production of pro-inflammatory cytokine interleukin-18 in lipopolysaccharide stimulated murine macrophage-like cells. Indian J Clin Biochem. 2015; 30(1):109-112.

33. Shishodia S. Molecular mechanisms of curcumin action: gene expression. Biofactors. 2013;39(1):37-55.

34. Yen FL, Wu TH, Tzeng CW, Lin LT, Lin CC. Curcumin nanoparticles improve the physicochemical properties of curcumin and effectively enhance its antioxidant and antihepatoma activities. J Agric Food Chem. 2010;58(12):7376-7382.

35. de Abreu Costa L, Henrique Fernandes Ottoni M, Dos Santos M, et al. Dimethyl sulfoxide (DMSO) decreases cell proliferation and TNF- $\alpha$, IFN- $\gamma$, and IL-2 cytokines production in cultures of peripheral blood lymphocytes. Molecules. 2017;22(11):1789.

36. Maral S, Albayrak M, Pala C, Yildiz A, Sahin O, Ozturk HB. Dimethyl sulfoxide-induced tonic-clonic seizure and cardiac arrest during infusion of autologous peripheral blood stem cells. Cell Tissue Bank. 2018; 19(4):831-832.

37. Morris C, de Wreede L, Scholten M, et al. Should the standard dimethyl sulfoxide concentration be reduced? Results of a European Group for Blood and Marrow Transplantation prospective noninterventional study on usage and side effects of dimethyl sulfoxide. Transfusion. 2014;54(10):2514-2522.

38. Windrum P, Morris TC, Drake MB, Niederwieser D, Ruutu T, Subcommittee E, EBMT Chronic Leukaemia Working Party Complications Subcommittee. Variation in dimethyl sulfoxide use in stem cell transplantation: a survey of EBMT centres. Bone Marrow Transplant. 2005;36(7):601-603.

39. Yen FL, Tsai MH, Yang CM, et al. Curcumin nanoparticles ameliorate ICAM-1 expression in TNF- $\alpha$-treated lung epithelial cells through $\mathrm{p} 47$ (phox) and MAPKs/AP-1 pathways. PLoS One. 2013;8(5):e63845.

40. Thurlow LR, Hanke ML, Fritz T, et al. Staphylococcus aureus biofilms prevent macrophage phagocytosis and attenuate inflammation in vivo. J Immunol. 2011;186(11):6585-6596.

41. Saqib U, Sarkar S, Suk K, Mohammad O, Baig MS, Savai R. Phytochemicals as modulators of M1-M2 macrophages in inflammation. Oncotarget. 2018;9(25):17937-17950.

42. Mantovani A, Sozzani S, Locati M, Allavena P, Sica A. Macrophage polarization: tumor-associated macrophages as a paradigm for polarized M2 mononuclear phagocytes. Trends Immunol. 2002;23(11):549-555.

43. Bottner F, Wegner A, Winkelmann W, Becker K, Erren M, Götze C. Interleukin-6, procalcitonin and TNF-alpha: markers of peri-prosthetic infection following total joint replacement. J Bone Joint Surg Br. 2007;89(1):94-99.

44. Shahi A, Parvizi J. The role of biomarkers in the diagnosis of periprosthetic joint infection. EFORT Open Rev. 2016;1(7):275-278.

45. Mittal M, Siddiqui MR, Tran K, Reddy SP, Malik AB. Reactive oxygen species in inflammation and tissue injury. Antioxid Redox Signal. 2014; 20(7):1126-1167.

46. González JF, Hahn MM, Gunn JS. Chronic biofilm-based infections: skewing of the immune response. Pathog Dis. 2018;76(3).

47. Kerksiek KM, Pamer EG. T cell responses to bacterial infection. Curr Opin Immunol. 1999;11(4):400-405.

48. Ziegler C, Goldmann O, Hobeika E, Geffers R, Peters G, Medina E. The dynamics of T cells during persistent Staphylococcus aureus infection: from antigen-reactivity to in vivo anergy. EMBO Mol Med. 2011 3(11):652-666.

49. Medina E, Hartl D. Myeloid-derived suppressor cells in infection: a general overview. J Innate Immun. 2018:1-7.

50. Heim CE, Vidlak D, Odvody J, Hartman CW, Garvin KL, Kielian T. Human prosthetic joint infections are associated with myeloid-derived suppressor cells (MDSCs): Implications for infection persistence. J Orthop Res. 2018;36(6):1605-1613.

51. Lew DP, WaldvogelFA. Osteomyelitis. Lancet. 2004;364(9431):369-379. 
52. Dudek-Wicher RK, Junka A, Bartoszewicz M. The influence of antibiotics and dietary components on gut microbiota. Prz Gastroenterol. 2018;13(2):85-92.

53. Tamadaho RSE, Hoerauf A, Layland LE. Immunomodulatory effects of myeloid-derived suppressor cells in diseases: Role in cancer and infections. Immunobiology. 2018;223(4-5):432-442.

54. Dorhoi A, Du Plessis N. Monocytic myeloid-derived suppressor cells in chronic infections. Front Immunol. 2017;8:1895.

55. Zhou Y, Zhang T, Wang X, et al. Curcumin modulates macrophage polarization through the inhibition of the Toll-like receptor 4 expression and its signaling pathways. Cell Physiol Biochem. 2015;36(2):631-641.

56. Gao S, Zhou J, Liu N, et al. Curcumin induces M2 macrophage polarization by secretion IL-4 and/or IL-13. J Mol Cell Cardiol. 2015;85: 131-139.
57. Pastuła A, Marcinkiewicz J. Myeloid-derived suppressor cells: a doubleedged sword? Int J Exp Pathol. 2011;92(2):73-78.

58. Condamine T, Gabrilovich DI. Molecular mechanisms regulating myeloid-derived suppressor cell differentiation and function. Trends Immunol. 2011;32(1):19-25.

59. Jiang M, Chen J, Zhang W, et al. Interleukin-6 trans-signaling pathway promotes immunosuppressive myeloid-derived suppressor cells via suppression of suppressor of cytokine signaling 3 in breast cancer. Front Immunol. 2017;8:1840.

60. Zambrano LMG, Brandao DA, Rocha FRG, et al. Local administration of curcumin-loaded nanoparticles effectively inhibits inflammation and bone resorption associated with experimental periodontal disease. Sci Rep. 2018;8(1):6652. 


\section{Supplementary material}

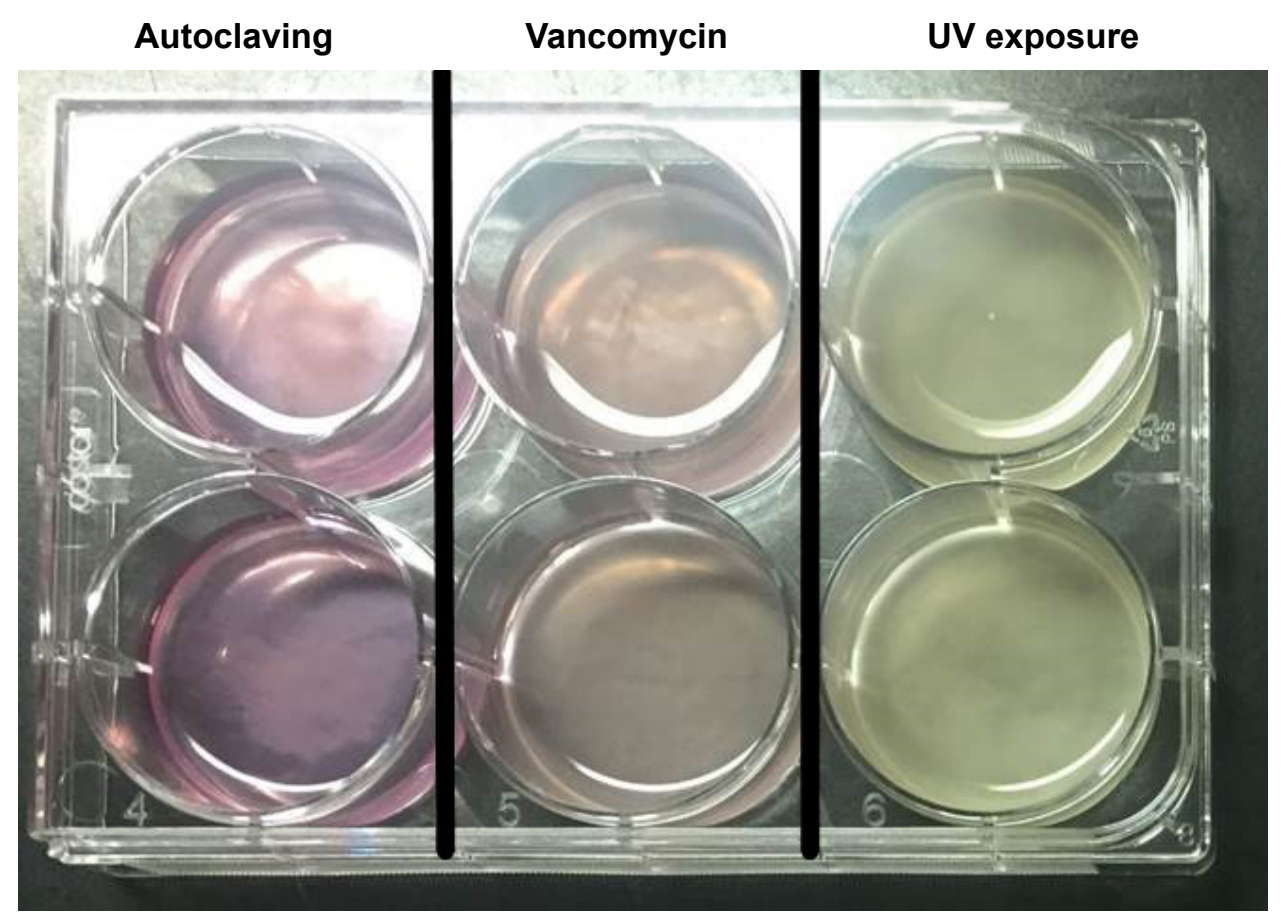

Figure SI The supplementary data demonstrated that the autoclaving method for biofilm is the optimal method in our system.

Notes: S. aureus and its biofilm were collected by centrifugation at 3,900 $\mathrm{g}$ for 15 minutes, and then the pellet was sterilized by autoclaving or pre-treatment with vancomycin or UV for 12 hours (from lane I to lane 3) before adding to BMC culture. Both pre-treatment with vancomycin (lane 2) and UV for I2 hours (lane 3) failed to reduce the $S$. aureus growth rate. If the collected pellet was not autoclaved, the growth of $S$. aureus was rapid and resulted in the death of BMCs within 48 hours.

Abbreviations: BMCs, bone marrow cells; S. aureus, Staphylococcus aureus.

International Journal of Nanomedicine

Dovepress

\section{Publish your work in this journal}

The International Journal of Nanomedicine is an international, peerreviewed journal focusing on the application of nanotechnology in diagnostics, therapeutics, and drug delivery systems throughou the biomedical field. This journal is indexed on PubMed Central, MedLine, CAS, SciSearch ${ }^{\circledR}$, Current Contents ${ }^{\circledR} /$ Clinical Medicine,
Journal Citation Reports/Science Edition, EMBase, Scopus and the Elsevier Bibliographic databases. The manuscript management system is completely online and includes a very quick and fair peer-review system, which is all easy to use. Visit http://www.dovepress.com/ testimonials.php to read real quotes from published authors. 